\title{
Otras variables que inciden en la competencia lectora en graduandos de Baja Verapaz, Guatemala
}

\author{
Alfredo Primero Camajá \\ Alfredok.amaja@gmail.com \\ https://orcid.org/0000-0003-1475-5693 \\ Facultad de Ingeniería en Sistemas, \\ Universidad Mariano Gálvez de Guatemala \\ Guatemala - Guatemala
}

\section{RESUMEN}

El presente estudio expone la percepción que tienen los docentes que laboran en el nivel medio en el departamento de Baja Verapaz, Guatemala, respecto a quien tiene la responsabilidad de desarrollar las competencias lectoras en los alumnos. Este un trabajo de investigación de tipo cuantitativo transversal descriptivo, analiza la opinión de 126 docentes. El objetivo general fue identificar las prácticas y percepciones que tienen los docentes de nivel medio que trabajan con los alumnos graduandos respecto a la implementación de las estrategias de lectura y de quién es la responsabilidad de llevarlas a cabo en el salón de clases. El estudio reflejó que son los docentes de Comunicación y Lenguaje, y Literatura los que se autoidentifican como los responsables de la implementación de la lectura en el establecimiento, de igual manera así lo ven su colega que imparten las otras áreas, es decir, los demás docentes se sienten ajenos a desarrollar las habilidades lectoras en los alumnos, teniendo una visión parcializada de su labor docente.

Palabras clave: comprensión; lectura; estrategia. 


\title{
Perception of middle school teachers in Baja Verapaz, Guatemala about who should develop reading skills in students
}

\begin{abstract}
This study exposes the perception of teachers who work at the secondary level in the department of Baja Verapaz, Guatemala, regarding who has the responsibility of developing reading skills in students. This descriptive cross-sectional quantitative research work analyzes the opinion of 126 teachers. The general objective was to identify the practices and perceptions that high school teachers who work with graduating students have regarding the implementation of reading strategies and who is responsible for carrying them out in the classroom. The study showed that the Communication and Language and Literature teachers are the ones who self-identify as those responsible for the implementation of reading in the establishment, in the same way that their colleagues who teach the other areas see it, that is, the other teachers feel oblivious to developing reading skills in students, having a biased view of their teaching work.
\end{abstract}

Keywords: comprehension; reading; strategy.

Artículo recibido: 15 enero 2022 Aceptado para publicación: 08 febrero 2022 Correspondencia: Alfredok.amaja@gmail.com Conflictos de Interés: Ninguna que declarar 


\section{INTRODUCCIÓN}

El presente estudio expone la percepción que tienen los docentes que trabajan los diversos cursos en el ciclo de educación diversificada y las habilidades lectoras que manifiestan desarrollan con los alumnos en el departamento de Baja Verapaz, Guatemala. En los datos que reporta el Ministerio de Educación, se tiene el número de periodos de lectura que los docentes le dedican a nivel semanal, y se analizan algunas variables de los docentes que imparten el curso de Lenguaje y Comunicación y Literatura, pero se desconoce la opinión de los docentes que trabajan en las otras áreas curriculares y de cómo éstos implementan las habilidades de lectura, por esto, este estudio buscan completar, aportar a los datos que el Ministerio de Educación presentan en sus distintos informes. También el estudio es importante porque "el 27.19\% de los graduandos de Baja Verapaz, alcanzan el criterio de la competencia lectora que se requiere". (Quim, 2020). Ante esta situación es necesario analizar las razones de este bajo resultado, puesto que se busca que las instituciones de servicio educativo, sean sujetas de investigación para saber que están haciendo bien, y que aspectos necesitan mejorar, aunado a lo que la (UNESCO, 2017) pide al referirse a lo siguiente: "Rendir cuentas significa ser capaces de actuar cuando algo va mal, por medio de la política, las leyes y la promoción, inclusive a través de los defensores de los ciudadanos para proteger los derechos de estos. Tenemos necesidad de mecanismos generales más sólidos para consagrar y hacer cumplir el derecho a la educación y exigir a todos los gobiernos cuentas de sus compromisos, incluidos los donantes". La investigación en el ámbito social no está llamada únicamente a describir las situaciones de la población, sino que aporta las herramientas de comprensión y posible intervención en tales situaciones. A continuación, el Ministerio de Educación, "En el marco de referencia de las pruebas nacionales, se localiza la siguiente tabla como una descripción de las estrategias que evalúa la prueba de lectura para estudiantes graduandos": 


\begin{tabular}{|c|c|c|c|}
\hline \begin{tabular}{c|} 
Nivel de \\
comprensión \\
lectora
\end{tabular} & $\begin{array}{c}\text { ¿Qué se } \\
\text { espera del } \\
\text { estudiante? }\end{array}$ & $\begin{array}{l}\text { Destreza o } \\
\text { estrategia } \\
\text { evaluada }\end{array}$ & Descripción de la destreza \\
\hline \multirow{6}{*}{ Literal } & \multirow{6}{*}{$\begin{array}{l}\text { Que } \\
\text { interprete el } \\
\text { sentido } \\
\text { exacto y } \\
\text { propio no } \\
\text { figurado de } \\
\text { las palabras } \\
\text { empleadas en } \\
\text { el texto } \\
\text { original. }\end{array}$} & $\begin{array}{l}\text { Sinónimo (estrategia } \\
\text { de vocabulario) }\end{array}$ & $\begin{array}{l}\text { Identificar la palabra que significa lo mismo que la } \\
\text { palabra resaltada en el texto, teniendo en cuenta el } \\
\text { contexto. }\end{array}$ \\
\hline & & $\begin{array}{l}\text { Antónimo (estrategia } \\
\text { de vocabulario) }\end{array}$ & $\begin{array}{l}\text { Encontrar la palabra que significa lo contrario que la } \\
\text { palabra resaltada en el texto, teniendo en cuenta el } \\
\text { contexto. }\end{array}$ \\
\hline & & $\begin{array}{l}\text { Detalle (destreza de } \\
\text { comprensión) }\end{array}$ & $\begin{array}{l}\text { Localizar información específica en diferentes tipos } \\
\text { de texto, para responder a preguntas directas } \\
\text { explícitas del texto o personaje. }\end{array}$ \\
\hline & & $\begin{array}{l}\text { Secuencia (destreza } \\
\text { de comprensión) }\end{array}$ & Identificar el orden cronológico de eventos. \\
\hline & & $\begin{array}{l}\text { Tema (destreza de } \\
\text { comprensión) }\end{array}$ & Expresar en una frase de qué trata el texto. \\
\hline & & $\begin{array}{l}\text { Hechos (destreza de } \\
\text { comprensión) }\end{array}$ & $\begin{array}{l}\text { Identificar la información falsa o verdadera que } \\
\text { aparece explícita en el texto. }\end{array}$ \\
\hline \multirow{9}{*}{ Inferencial } & \multirow{9}{*}{$\begin{array}{l}\text { Que deduzca } \\
\text { la } \\
\text { información } \\
\text { implicada } \\
\text { pero no } \\
\text { expresada en } \\
\text { el texto. }\end{array}$} & $\begin{array}{l}\text { Similitudes } \\
\text { diferencias } \\
\text { (destrezas } \\
\text { cognitivas) }\end{array}$ & $\begin{array}{l}\text { Fijar la atención en dos o más objetos para descubrir } \\
\text { diferencias y similitudes. }\end{array}$ \\
\hline & & $\begin{array}{lr}\text { Clave de } & \text { contexto } \\
\text { (estrategia } & \text { de } \\
\text { vocabulario) } & \end{array}$ & $\begin{array}{l}\text { Identificar el significado de una palabra usando } \\
\text { como pistas o indicios textuales, otras palabras, } \\
\text { frases u oraciones del texto. }\end{array}$ \\
\hline & & $\begin{array}{l}\text { Predicción } \\
\text { (estrategia } \\
\text { comprensión) }\end{array}$ & $\begin{array}{l}\text { Anticipar lo que puede ocurrir o suceder en la } \\
\text { narración, según una situación o acontecimiento } \\
\text { dado al lector. }\end{array}$ \\
\hline & & $\begin{array}{l}\text { Lenguaje figurado } \\
\text { (destreza cognitiva) }\end{array}$ & $\begin{array}{l}\text { Analizar locuciones para deducir su significado } \\
\text { connotativo }\end{array}$ \\
\hline & & $\begin{array}{l}\text { Idea principal } \\
\text { implícita (destreza de } \\
\text { comprensión) }\end{array}$ & Encontrar la idea que mejor resume el texto. \\
\hline & & $\begin{array}{l}\text { Causa-efecto } \\
\text { (destreza cognitiva) }\end{array}$ & $\begin{array}{l}\text { Identificar el fundamento originado por algo dentro } \\
\text { de un contexto. }\end{array}$ \\
\hline & & $\begin{array}{l}\text { Conclusión (destreza } \\
\text { de comprensión) }\end{array}$ & $\begin{array}{l}\text { Formular con una idea final lo expuesto en el texto } \\
\text { objetivamente. }\end{array}$ \\
\hline & & $\begin{array}{l}\text { Generalización } \\
\text { (estrategia de } \\
\text { comprensión) }\end{array}$ & $\begin{array}{l}\text { Abstraer lo que es común y esencial a muchas cosas, } \\
\text { para formar el concepto general de las ideas } \\
\text { relacionadas con esa abstracción. }\end{array}$ \\
\hline & & $\begin{array}{l}\text { Hipótesis (estrategia } \\
\text { de comprensión) }\end{array}$ & $\begin{array}{l}\text { Formular una suposición de algo posible a partir del } \\
\text { texto. }\end{array}$ \\
\hline \multirow{3}{*}{ Crítico } & \multirow{3}{*}{$\begin{array}{l}\text { Que exprese } \\
\text { y formule un } \\
\text { juicio } \\
\text { relacionado } \\
\text { con las ideas } \\
\text { contenidas en } \\
\text { el texto. }\end{array}$} & $\begin{array}{l}\text { Intención } \\
\text { comunicativa del } \\
\text { texto (destreza de } \\
\text { comprensión) }\end{array}$ & $\begin{array}{l}\text { Analizar el texto identificando el punto de vista del } \\
\text { autor, dependiendo de la perspectiva desde la que } \\
\text { expone el tema. }\end{array}$ \\
\hline & & $\begin{array}{l}\text { Opinión (destreza de } \\
\text { comprensión) }\end{array}$ & $\begin{array}{l}\text { Identificar un hecho comprobable descrito en el texto } \\
\text { y distinguirlo de una opinión (emitir un juicio que se } \\
\text { forma acerca de un tema cuestionable). }\end{array}$ \\
\hline & & $\begin{array}{l}\text { Resumen (estrategia } \\
\text { de comprensión) }\end{array}$ & $\begin{array}{l}\text { Reducir a términos breves y esenciales los párrafos } \\
\text { de un texto. }\end{array}$ \\
\hline
\end{tabular}

(Del Valle, 2017) 
El Ministerio de Educación ha impulsado estrategias de apoyo a los docentes de Comunicación y Lenguaje para el desarrollo de cada una de las habilidades antes descritas, tanto para que los alumnos las refuercen a través del proyecto Prueba T, así como de cartillas como aprender del error, dirigido a docentes "como un instrumento para reflexionar en torno a los resultados de las evaluaciones" (Digeduca, 2015) y que los estudiantes generen aprendizajes a través de los errores que no permitieron elevar el logro de la lectura. Sin embargo, hacen falta más esfuerzos para que el desarrollo de la competencia lectora la alcancen los estudiantes. La competencia lectora ha evolucionado a lo largo del tiempo. Este, sumando al de aprendizaje continuo o permanente, da como resultado la consideración de que las competencias lectoras o se adquieren solo en los primeros años de escuela: se van desarrollando a lo largo de la vida y se modifican según los distintos contextos en que se desenvuelve la vida de las personas. Para el Programa Internacional de Evaluación de Estudiantes -PISA- por sus siglas en inglés- la "competencia lectora es comprender, utilizar, reflexionar y comprometerse con textos escritos, para alcanzar los propios objetivos, desarrollar el conocimiento y potencial personales, y participar en la sociedad" OCDE.2012. Citado en (Del Valle, 2017). La competencia es "la capacidad o disposición que ha desarrollado una persona para afrontar y dar solución a problemas de la vida cotidiana y generar nuevos conocimientos" (MINEDUC, 2012). Los resultados en lectura de los estudiantes graduandos inciden en la vida cotidiana y refleja el rendimiento en el grado inmediato superior. Las competencias para la vida son "aquellas competencias clave o básicas que todo estudiante deberá dominar al término de la secundaria o nivel medio y que le permitan proseguir los estudios, recibir una formación profesional e integrarse socialmente sin riesgo de exclusión" (USAID, 2009). El desarrollo de las competencias de lectura no debe delimitarse al área curricular de Comunicación y Lenguaje, debe ser abordada como una acción colectiva, y se hace necesario identificar entre competencias explícitas y competencias implícitas. Las competencias explícitas se identifican literalmente en el área curricular de Comunicación y Lenguaje del Currículo Nacional Base de cada carrera del ciclo diversificado, aunque existen carreras que no tienen el área curricular en mención, se identifica en la segunda competencia del área curricular de Comunicación y Lenguaje L1 de la carrera de Bachillerato en Ciencias y Letras con Orientación en Educación explícitamente dice "utiliza la lectura de obras de la literatura de los Pueblos 
del mundo para generar opiniones" Las competencias implícitas de la lectura se implementan durante la vida escolar y en las diferentes áreas curriculares de manera transversal. La lectura es una herramienta para la construcción del conocimiento, nadie asciende al grado inmediato superior si no lee. Una muestra de las competencias implícitas de la lectura están presentes en matemática en donde se evidencia el uso de las estrategias para "resolver problemas, pueden estimar, predecir, hacer conclusiones, evaluar y usar la información eficientemente" (Cotto, 2012). Identificadas en la serie de cuadernillos pedagógicos de la evaluación a la acción de la Digeduca de sexto grado del nivel primario. Tal como lo expresan los autores citados, adquirir la competencia lectora es un proceso que va más allá del simple hecho de decodificar, implica interactuar con las ideas del autor, hasta llegar a construir un significado propio aunado a la propia experiencia, por eso se dice, que dos personas que comprenden un texto, lo comprenden de manera distinta, pues cada quien posee una experiencia distinta. En el área curricular de Comunicación y Lenguaje se describen algunas competencias de lectura y escritura que los alumnos deben de desarrollar, pero existen carreras que no tienen Comunicación y Lenguaje en su pensum de estudios. Por esto, es importante insistir que independientemente del curso que se desarrolle, cada docente debe implementar procesos de análisis, síntesis, identificación de la idea central, establecer diferencias y semejanzas, causas y efectos, solo por mencionar unas cuantas habilidades que le permitirían al estudiante fortalecer las habilidades de lectura en cada uno de los textos que tiene la oportunidad de leer, por ejemplo en el curso de Estudios Socioeconómicos de Guatemala, podría comparar los distintos puntos de vista de los autores que abordan un tema, establecer diferencias y semejanzas, analizar cuando es un hecho lo que aparece en un párrafo y cuando es una opinión del propio autor. Acá radica la importancia de este estudio que permite identificar las prácticas y percepciones en relación a la lectura, a través de la siguiente pregunta de investigación: ¿Cuáles son las prácticas y percepciones que tienen los docentes de nivel medio que trabajan con graduandos respecto a la implementación de la lectura? De donde se generan, las siguientes preguntas específicas que determinan el curso de la investigación: ¿Cuáles son las estrategias de lectura que utilizan los docentes independientemente del curso que imparten? ¿Quiénes son los docentes responsables de impulsar la lectura dentro del establecimiento? 


\section{ESTRATEGIAS METODOLÓGICAS, MATERIALES Y MÉTODOS}

Los centros educativos del nivel medio del ciclo diversificado participantes en el estudio pertenecen a los municipios de Salamá, San Miguel Chicaj, Rabinal, Cubulco, Granados, Santa Cruz El Chol, San Jerónimo y Purulhá, siendo el 100\% de los municipios del departamento de Baja Verapaz, Guatemala. La unidad de análisis fueron los docentes de graduandos con perfil académico universitario a nivel de profesorado, con licenciatura en educación y algunos con postgrado siempre en educación. El enfoque de la investigación es cuantitativo, puesto se trabajó con datos cuantitativos en una encuesta que le aplicó a los docentes, de igual manera se cataloga como transversal, pues únicamente se hizo una medición de las variables de estudio y finalmente de tipo descriptivo. Según (Achaerandio, 2012) “La investigación descriptiva...examina sistemáticamente y analiza la conducta humana personal y social en condiciones naturales, y en los distintos ámbitos (en la familia, en el sistema educativo formal, en el trabajo, en lo social, lo económico, lo político, lo religioso, etc.). Como toda investigación, la descriptiva busca la solución de algún problema, o alcanzar una meta del conocimiento. Suele comenzar con el estudio y análisis de la situación presente". La muestra fue por conveniencia, en donde participaron 126 docentes, de los municipios ya mencionados. Según (Hernández-Sampieri, 2014) haciendo referencia a (Battaglia, 2008a), describe que "las muestras por conveniencia: estas muestras están formadas por los casos disponibles a los cuales tenemos acceso". Los criterios de la selección fueron docentes que están laborando en último grado del ciclo diversificado, siendo en su mayoría del sector oficial y en los municipios de poca población estudiantil se tomaron algunos establecimientos del sector privado. La estrategia de recopilación de la información fue una convocatoria en donde asistieron los docentes y se les hizo ver el objetivo de la investigación, luego procedieron a llenar el cuestionario. El instrumento de recolección de la información fue creado exclusivamente para docentes tomando como referente el cuestionario aplicado a directores de los centros educativos del nivel medio ciclo diversificado en el año 2018, elaborado por la Digeduca/ Ministerio de Educación, de la cual se tomaron 7 ítems y se crearon 6 ítems nuevos para responder a los objetivos del presente estudio. Algunas de las variables de estudio fueron: el uso de estrategias lectura, la implementación de la lectura y la especialidad del curso. La información fue analizada por medio de SPSS software/IMB. Finalmente según 
(Hernández-Sampieri, 2014). "La limitación en este tipo de investigación es que los resultados se aplican nada más a la muestra en sí o a muestras similares en tiempo y lugar (transferencia de resultados); No son generalizables a una población ni interesa esta extrapolación". Tal como ya se expresó lo valioso de este estudio es que puede ser la base para una investigación de mayor envergadura en el país.

\section{RESULTADOS Y DISCUSIÓN}

A los docentes se les consultó si impulsan la lectura dentro del establecimiento, si la respuesta es afirmativa que indique el área curricular que tienen a su cargo. Además, en una tercera pregunta se les solicitó a que indicaran quién es el responsable de impulsar la lectura actualmente en el centro educativo. En la primera y segunda columna de la siguiente tabla, se puede identificar los 126 docentes que afirmaron implementar la lectura en el área o subárea curricular que tiene a su cargo. En la columna de área y subárea curricular que tiene a su cargo se pueden leer las áreas de acuerdo al pensum de estudios de cada carrera que intervinieron en el ejercicio. Para efecto de análisis el subárea Literatura se desglosó del área de Comunicación y Lenguaje, y se agruparon las áreas curriculares: humanísticas, matemática y tecnología, y otras especialidades, cubriendo así todas las áreas que indicaron los docentes. La siguiente tabla responde a la pregunta ¿Actualmente quién impulsa la lectura dentro del establecimiento? Cada docente participante tuvo la oportunidad de marcar tres opciones: docente de literatura, docente de comunicación y lenguaje, todo el personal. El 73.1\% de los docentes de Literatura aceptaron la responsabilidad de implementar la lectura dentro del establecimiento, mientras que el $41.7 \%$ de los docentes de Comunicación y Lenguaje también aceptaron la responsabilidad, los docentes de otras áreas curriculares se perciben con baja responsabilidad de llevar a cabo esta acción.

Tabla No. 1 ¿Actualmente quién impulsa la lectura dentro del establecimiento?

\begin{tabular}{l|c|c|c|c}
\hline Área curricular que tiene a su cargo & $\begin{array}{c}\text { Cantidad } \\
\text { de } \\
\text { docentes }\end{array}$ & $\begin{array}{c}\text { Docente de } \\
\text { literatura }\end{array}$ & $\begin{array}{c}\text { Docente de } \\
\text { comunicación } \\
\text { y lenguaje }\end{array}$ & $\begin{array}{c}\text { Todo el } \\
\text { personal }\end{array}$ \\
\hline 1. Comunicación y Lenguaje & 12 & $33.3 \%$ & $41.7 \%$ & $25.0 \%$ \\
\hline 2. Literatura & 26 & $73.1 \%$ & $7.7 \%$ & $15.4 \%$ \\
\hline 3. Matemática y tecnología & 18 & $44.4 \%$ & $27.8 \%$ & $27.8 \%$ \\
\hline 4. Administración & 3 & $0.0 \%$ & $33.3 \%$ & $0.0 \%$ \\
\hline 5. Humanísticas & 47 & $38.3 \%$ & $21.3 \%$ & $31.9 \%$ \\
\hline 6. Otras especialidades & 20 & $30.0 \%$ & $40.0 \%$ & $10.0 \%$ \\
\hline
\end{tabular}

Fuente: Cuestionario aplicado a docentes de nivel medio del departamento de Baja Verapaz. 
Las siguientes tablas muestran las respuestas de los docentes en cuanto al uso de las estrategias de lectura en una clase regular. A los docentes se les presentó las dieciocho estrategias de lectura que se espera que los estudiantes graduandos tengan las competencias cuando egresen del Sistema de Educativo Nacional. Cada docente marcó lo que solicita a sus estudiantes en el aula de clase regular. A continuación, se muestran las respuestas clasificadas en tres categorías.

Tabla No. 2 Principales estrategias utilizadas

\begin{tabular}{|c|c|c|c|c|c|c|c|c|}
\hline $\begin{array}{c}\text { Área Curricular que tiene a } \\
\text { su cargo }\end{array}$ & $\begin{array}{c}\text { Cantidad } \\
\text { de } \\
\text { docentes }\end{array}$ & 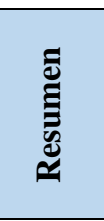 & 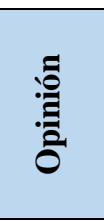 & 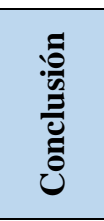 & 氖 & 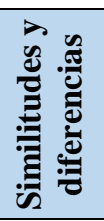 & 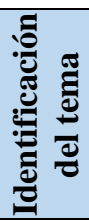 & 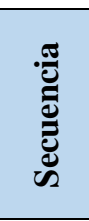 \\
\hline 1.Comunicación y Lenguaje & 12 & $75 \%$ & $83 \%$ & $75 \%$ & $75 \%$ & $58 \%$ & $58 \%$ & $75 \%$ \\
\hline 2. Literatura & 26 & $85 \%$ & $77 \%$ & $73 \%$ & $81 \%$ & $77 \%$ & $69 \%$ & $65 \%$ \\
\hline 3. Matemática y tecnología & 18 & $72 \%$ & $72 \%$ & $78 \%$ & $67 \%$ & $56 \%$ & $39 \%$ & $56 \%$ \\
\hline 4. Administración & 3 & $100 \%$ & $100 \%$ & $100 \%$ & $100 \%$ & $33 \%$ & $67 \%$ & $33 \%$ \\
\hline 5. Humanísticas & 47 & $85 \%$ & $83 \%$ & $87 \%$ & $79 \%$ & $62 \%$ & $66 \%$ & $51 \%$ \\
\hline 6. Otras especialidades & 20 & $75 \%$ & $70 \%$ & $65 \%$ & $85 \%$ & $60 \%$ & $60 \%$ & $55 \%$ \\
\hline
\end{tabular}

Fuente: Cuestionario aplicado a docentes de nivel medio del departamento de Baja Verapaz.

En la segunda categoría se evidencia las estrategias usado en nivel intermedio por los docentes, sin embargo, los docentes que hacen más uso de estas habilidades, son los docentes de Comunicación y Lenguaje, también los docentes de Literatura.

Tabla No. 3 Estrategias de uso intermedio

\begin{tabular}{|c|c|c|c|c|c|c|c|c|c|}
\hline $\begin{array}{l}\text { Área Curricular que tiene a } \\
\text { su cargo }\end{array}$ & $\begin{array}{c}\text { Cantidad } \\
\text { de } \\
\text { docentes }\end{array}$ & 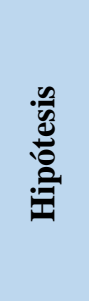 & 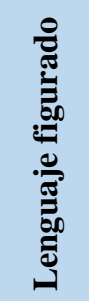 & 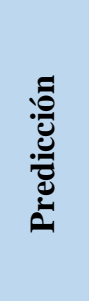 & 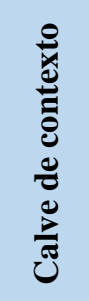 & 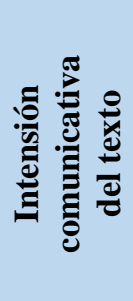 & 窇 & 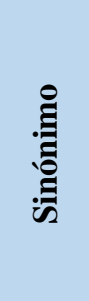 & 总 \\
\hline 1.Comunicación y Lenguaje & 12 & $50 \%$ & $58 \%$ & $58 \%$ & $67 \%$ & $42 \%$ & $33 \%$ & $67 \%$ & $67 \%$ \\
\hline 2. Literatura & 26 & $27 \%$ & $31 \%$ & $42 \%$ & $12 \%$ & $50 \%$ & $62 \%$ & $62 \%$ & $58 \%$ \\
\hline 3.Matemática y tecnología & 18 & $33 \%$ & $17 \%$ & $6 \%$ & $22 \%$ & $17 \%$ & $22 \%$ & $11 \%$ & $22 \%$ \\
\hline 4. Administración & 3 & $67 \%$ & $33 \%$ & $33 \%$ & $33 \%$ & $67 \%$ & $33 \%$ & $0 \%$ & $0 \%$ \\
\hline 5. Humanísticas & 47 & $34 \%$ & $15 \%$ & $19 \%$ & $21 \%$ & $38 \%$ & $23 \%$ & $38 \%$ & $26 \%$ \\
\hline 6. Otras especialidades & 20 & $20 \%$ & $15 \%$ & $25 \%$ & $30 \%$ & $20 \%$ & $30 \%$ & $35 \%$ & $30 \%$ \\
\hline
\end{tabular}

Fuente: Cuestionario aplicado a docentes de nivel medio del departamento de Baja Verapaz. 
Y, en la última categoría se encuentran el uso de las estrategias: generalización, causas y efecto, y detalle. Agrupa el menor porcentaje de docentes que la trabajan en una clase regular.

Tabla No. 4 Estrategias de poco uso

\begin{tabular}{|c|c|c|c|c|}
\hline Área Curricular que tiene a su cargo & $\begin{array}{l}\text { Cantidad de } \\
\text { docentes }\end{array}$ & 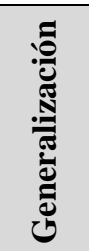 & 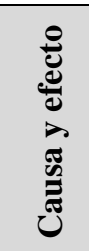 & 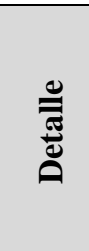 \\
\hline 1. Comunicación y Lenguaje & 12 & $33 \%$ & $42 \%$ & $33 \%$ \\
\hline 2. Literatura & 26 & $35 \%$ & $42 \%$ & $23 \%$ \\
\hline 3. Matemática y tecnología & 18 & $28 \%$ & $28 \%$ & $11 \%$ \\
\hline 4. Administración & 3 & $33 \%$ & $67 \%$ & $0 \%$ \\
\hline 5. Humanísticas & 47 & $11 \%$ & $23 \%$ & $21 \%$ \\
\hline 6. Otras especialidades & 20 & $20 \%$ & $10 \%$ & $20 \%$ \\
\hline
\end{tabular}

Fuente: Cuestionario aplicado a docentes de nivel medio del departamento de Baja Verapaz.

El uso de estrategias de lectura permite mejor comprensión, tomando en cuenta que "la lectura puede ser vista como una actividad para obtener información de un código lingüístico a través del canal visual” (Flórez, 2006). La información que se adquiere a través del uso de estrategias de lectura, permite el logro de las competencias de cada área o sub área curricular.

\section{CONCLUSIONES}

Las prácticas y percepciones que tienen los docentes de nivel medio que trabajan con graduandos respecto a la implementación de la lectura, muestra que los docentes que imparten el área curricular de Comunicación y Lenguaje, y Literatura asumen la responsabilidad de impulsar la lectura dentro del establecimiento, mientras que los demás docentes de otras áreas curriculares lo perciben asimismo como ajenos a esta actividad.

En el análisis de las estrategias de lectura que utiliza el docente en el desarrollo de una clase regular en el aula, se concluye que hacen uso de las dieciocho estrategias de lectura, independientemente del área curricular que el docente tiene a su cargo, sin embargo, habrá que indagar en una futura investigación, cómo desarrollan cada una de estas estrategias a través de una actividad práctica, acatando lo que sugiere (Tejedor, 2018) "Desde mi punto de vista, sería un gran avance en la calidad de la investiga el intento de aproximarnos a la medida de conductas reales, a través de estrategias (registros) de observación o pruebas de "saber hacer". En temas como la evaluación de competencias, por ejemplo, nos 
proporcionaría datos muchos más reales que los que obtenemos por opinión de los encuestados; datos más reales con mayor potencial para orientar los procesos formativos consecuentes".

Se establecen tres categorías del uso de las estrategias de lectura, en la primera categoría se encuentran las estrategias de mayor uso, siendo el resumen, opinión, conclusión, idea principal, similitudes y diferencias, identificación del tema y secuencia. En la segunda categoría se encuentran las estrategias que tienen uso intermedio, siendo la hipótesis, lenguaje figurado, predicción, clave de contexto, intención comunicativa del texto, hecho, sinónimo y antónimo. En la tercera categoría se encuentran las estrategias de menor uso, siendo la generalización, causas y efecto, y detalle.

El $100 \%$ de los docentes impulsan la lectura dentro del establecimiento independiente del área curricular que tienen a cargo. En el área de Comunicación y Lenguaje tiene un mayor uso las estrategias establecidas en segunda categoría que está sobre el 50\%, siendo la hipótesis, lenguaje figurado, predicción, clave de contexto, intención comunicativa del texto, hecho, sinónimo y antónimo.

\section{REFERENCIAS BILBIOGRAFICAS}

Achaerandio, L. (2012). Iniciación a la Práctica de la Investigación. Guatemamala: Magna Terra Editores.

Cotto, E. \&. (2012). Lectura matemática. Guatemala: Divulgación, Issue 3. Digeduca/MINEDUC.

Del Valle, M. e. (2017). Ciclo de Construcción de una Prueba Estandarizada. Marco de Referencia de las Pruebas Nacionales. Guatemala: Dirección General de Evaluación e Investigación Educativa. Departamento de Desarrollo de Pruebas Monoligües.

Flórez, R. e. (2006). El Aprendizaje en la escuela: el lugar de la lectura y escritura. Educación y Educadores, 9(1), 117-133.

Hernández-Sampieri, R. (2014). Metodología de la Investigación (6ta. ed.). México: McGrawHill.

MINEDUC. (2012). Curriculum Nacional Base de Bachillerato en Ciencias y Letras con orientación en Educación. Guatemala: Digecur.

Quim, M. (2020). Informe Nacional de Graduandos 2019. Guatemala: Dirección General de Evaluación e Investigación Educativa, Ministerio de Educación. 
Sampieri, R. H. (2014). Metodologia de la Investigacion (sexta ed.). Mexico: McGRAWHILL / INTERAMERICANA EDITORES, S.A. DE C.V.

Tejedor, J. (2018). Investigación Educativa. La utilidad como criterio social de calidad. Revista de Investigación Educativa, 36(2), 315-330.

UNESCO. (15 de 2 de 2017). Redir cuentas en el ámbito de la educación: Cumplir nuestros compromisos. Resumen del Informe de Seguimiento de la Educación en el Mundo. Francia. . Recuperado el 6 de 1 de 2022, de www.unesco.org/gemreport

USAID. (2009). Competencias básicas para la vida Guatemala. Guatemala.

DIGEDUCA. (2015). Serie aprender el error. Guatemala.

Díaz Hernández, A. A. (2015). Informe Final del Ejercicio Profesional Supervisado, Realizado en el Instituto de Educación Básica Por Cooperativa de Enseñanza de San Miguel Chicaj, Baja Verapaz (Doctoral dissertation, CUNOR).

Pérez Rodríguez, O. A. (2015). Informe Final Del Ejercicio Profesional Supervisado, realizado en el Instituto Nacional De Educación Básica, Barrio Agua Caliente, Salamá, Baja Verapaz (Doctoral dissertation, CUNOR).

por la Educación, E. (2020). ¿ Cómo estamos en Educación?. Indicadores educativos de Guatemala y Prioridades para el Plan de Gobierno 2016, 3.

SAN CRISTÓBAL VERAPAZ, A. V. (2017). " ESTRATEGIAS METODOLOGICAS PARA LA COMPRENSIÓN LECTORA EN EL ÁREA DE COMUNICACIÓN Y LENGUAJE EN LOS ALUMNOS DE NIVEL MEDIO EN EL ÁREA URBANA DE.

DE DÍAZ, E. G. A. C. PROPUESTA METODOLÓGICA PARA LA COMPRENSIÓN LECTORA DE ALUMNAS DE $2^{\circ}$ BÁSICO DE UN ESTABLECIMIENTO PRIVADO DE LA CIUDAD DE GUATEMALA.

Gonzáles, L. (2018). Hábitos de Interés por la Lectura que tienen los Alumnos de la Escuela Normal Intercultural de Chiquimula (Doctoral dissertation, Tesis de Grado] Universidad Rafael Landívar, Zacapa, Guatemala).

de Instrumentos, S. D. D. (2009). Dirección General de Evaluación e Investigación Educativa.

Caniz, C., \& Sebastiana, M. L. (2020). Influencia de las estrategias de comprensión lectora en el aprendizaje de los estudiantes del ciclo básico: Estudio realizado 
con estudiantes de tercer grado básico de los Institutos por Cooperativa del municipio de Totonicapán (Doctoral dissertation, Universidad de San Carlos de Guatemala). 